\title{
EPIDEMIOLOGICAL EVALUATION OF PATIENTS WITH NEUROPSYCHIATRIC LUPUS IN A TERTIARY CENTER IN SÃO PAULO
}

Samila Costa Pinheiro Guerra Barros ${ }^{1, \star}$, Sérgio Mameri Meireles de Souza ${ }^{1}$, Diego Riveros Logrado ${ }^{1}$, Renê Lima Porto ${ }^{1}$, Dawton Yukito Torigoe ${ }^{1}$, Gabriela Araújo Munhoz ${ }^{1}$

1.Irmandade da Santa Casa de Misericórdia de São Paulo, São Paulo (SP), Brazil.

*Corresponding author: samilacpg@hotmail.com

\section{BACKGROUND}

Neuropsychiatric manifestations (NPS) are increasingly recognized in patients with systemic lupus erythematosus (SLE) and are responsible for considerable mortality in these patients. This study seeks to deepen epidemiological data and behavior of neuropsychiatric disease in our population. This series is of great importance for a better understanding of the disease, considering that its recognition, treatment and prognosis are notoriously difficult.

\section{METHODS}

Data obtained from electronic medical records of 301 patients with SLE regularly followed up between November 2019 and March 2020, at the outpatient clinic of the rheumatology department of a tertiary center in São Paulo. 43 patients with NPs impairment of the disease were selected, who met the criteria of Classification ACR/EULAR 2019 and SLICC 2012, in addition to the set of case definitions of the LESNP of the ACR 1999. Data related to clinical and laboratory manifestations, gender, current age, disease duration and its evolution, disease activity.

\section{RESULTS}

The prevalence of NPs in the total SLE sample was 14.3\%, with CNS involvement being $12.3 \%$ and PNS $1.3 \%$. There was concomitant central and peripheral involvement in two patients. Of the 43 patients, $86 \%$ were white (58.1\%), with a mean age at diagnosis of $24 \pm 11.2$ years and mean disease duration of $10 \pm 1.06$ years. The gender ratio was $6.2: 1 ; 86 \%$ had CNS damage, $9.3 \%$ PNS damage and $4.7 \%$ damage in both. Regarding immunological data, $97.1 \%$ had positive ANA and $53.5 \%$ anti-dsDNA positive. The most common clinical manifestations in the opening of SLE were cutaneous (90.7\%) and joint manifestations (90.7\%) followed by hematological manifestations (69.8\%) and (44.2\%). The NP event was detected early in $76.2 \%$ of the patients. Of these patients, $67.4 \%$ had NP involvement at the time of diagnosis. The most common NP manifestations were cerebrovascular diseases (40\%), followed by convulsion (37\%), psychosis (28\%) and cognitive dysfunction (26\%).

\section{CONCLUSION}

This study contributes to a better understanding of NP involvement in the Brazilian lupus population, allowing a comparison with the world population, identifying their similarities and differences. In addition, it provides a better understanding of this type of involvement in order to allow a more effective and rapid conduct in order to prevent harm.

\section{KEYWORDS}

Systemic lupus erythematosus, Neuropsychiatric, Epidemiology. 